\title{
Identification and Functional Analysis of ThADH1 and ThADH4 Genes Involved in Tolerance to Waterlogging Stress in Taxodium hybrid 'Zhongshanshan 406'
}

\author{
Lei Xuan ${ }^{1}$, Jianfeng Hua ${ }^{1, *}$, Fan Zhang ${ }^{1}$, Zhiquan Wang ${ }^{1}{ }^{\circledR}$, Xiaoxiao Pei ${ }^{1}$, Ying Yang ${ }^{1}$, Yunlong Yin ${ }^{1}$ \\ and David L. Creech ${ }^{2}$
}

Citation: Xuan, L.; Hua, J.; Zhang, F.; Wang, Z.; Pei, X.; Yang, Y.; Yin, Y.; Creech, D.L. Identification and Functional Analysis of ThADH1 and ThADH4 Genes Involved in Tolerance to Waterlogging Stress in Taxodium hybrid 'Zhongshanshan 406'. Genes 2021, 12, 225. https://doi.org/ 10.3390 /genes12020225

Academic Editor: Delphine Grivet Received: 13 December 2020

Accepted: 2 February 2021

Published: 4 February 2021

Publisher's Note: MDPI stays neutral with regard to jurisdictional claims in published maps and institutional affiliations.

Copyright: (c) 2021 by the authors. Licensee MDPI, Basel, Switzerland. This article is an open access article distributed under the terms and conditions of the Creative Commons Attribution (CC BY) license (https:/ / creativecommons.org/licenses/by/ $4.0 /)$.
1 Jiangsu Province Engineering Research Center of Taxodium Rich. Germplasm Innovation and Propagation, Institute of Botany, Jiangsu Province and Chinese Academy of Sciences (Nanjing Botanical Garden Mem, Sun Yat-Sen), Nanjing 210037, China; 13851991791@163.com (L.X.); mumizhongfeng@126.com (F.Z.); zhiquanjiejie@163.com (Z.W.); x18944051197@126.com (X.P.); yingyang@cnbg.net (Y.Y.); yiny1066@sina.com (Y.Y.)

2 Arthur Temple College of Forestry and Agriculture, Stephen F. Austin State University, Nacogdoches, TX 75962, USA; dcreech@sfasu.edu

* Correspondence: jfhua@cnbg.net; Tel.: +86-25-8434-7069

\begin{abstract}
The Taxodium hybrid 'Zhongshanshan 406' (T. hybrid 'Zhongshanshan 406') [Taxodium mucronatum Tenore $\times$ Taxodium distichum (L.). Rich] has an outstanding advantage in flooding tolerance and thus has been widely used in wetland afforestation in China. Alcohol dehydrogenase genes $(A D H s)$ played key roles in ethanol metabolism to maintain energy supply for plants in low-oxygen conditions. Two ADH genes were isolated and characterized-ThADH1 and ThADH4 (GenBank ID: AWL83216 and AWL83217—basing on the transcriptome data of T. hybrid 'Zhongshanshan 406' grown under waterlogging stress. Then the functions of these two genes were investigated through transient expression and overexpression. The results showed that the ThADH1 and ThADH4 proteins both fall under ADH III subfamily. ThADH1 was localized in the cytoplasm and nucleus, whereas ThADH4 was only localized in the cytoplasm. The expression of the two genes was stimulated by waterlogging and the expression level in roots was significantly higher than those in stems and leaves. The respective overexpression of ThADH1 and ThADH4 in Populus caused the opposite phenotype, while waterlogging tolerance of the two transgenic Populus significantly improved. Collectively, these results indicated that genes ThADH1 and ThADH4 were involved in the tolerance and adaptation to anaerobic conditions in $T$. hybrid 'Zhongshanshan 406 '.
\end{abstract}

Keywords: Taxodium hybrid 'Zhongshanshan 406'; ThADH; waterlogging; expression patterns

\section{Introduction}

In China, there are approximately 53.6 million hectares of wetlands. However, in recent decades, as a result of human activities, the ecological function of much of the wetlands has seriously deteriorated. Considering the Yangtze River Basin as an example, under the influence of urbanization and industrialization, nearly $60 \%$ of the wetlands in the middle and lower reaches of the Yangtze River are at risk of gradually disappearing, with the wetland ecosystem becoming seriously damaged [1]. The selection and cultivation of plants, especially flooding-tolerant trees, is particularly critical for the restoration and reconstruction of wetland ecosystems in China. It is reported that, compared with herbs, few woody plants can survive prolonged flooding, especially complete submergence [2,3]. Taxodium hybrid 'Zhongshanshan 406' ( $T$. hybrid 'Zhongshanshan 406') is an interspecific hybrid clone, produced from two Taxodium species, namely Taxodium mucronatum and Taxodium distichum [4], which showed a marked ability to withstand flooding. T. hybrid 'Zhongshanshan 406' has been widely planted in wetlands and lakes in China (including Poyang Lake in Jiangxi Province, Chao Lake in Anhui Province, Bagua Islet in Jiangsu 
Province) [5,6]. The experimental planting of T. hybrid 'Zhongshanshan 406' in the Three Gorges Reservoir of China showed an approximately $90 \%$ survival rate after the entire trees had been submerged for up to 122 days [5]. Recently, analysis of the flooding tolerance mechanism of $T$. hybrid 'Zhongshanshan 406' has become a research focus [7].

The ability of plants to withstand flooding and the recovery of growth after flooding depend on its carbohydrate reserves. After a long period of flooding stress, plants are finally in the state of energy starvation and the flood tolerant plants can maintain energy reserve by changing biochemical metabolic pathways [2]. In the flooding environment, the energy to maintain plant vitality mainly relies on the ethanol metabolic pathway in glycolysis to degrade glucose and glycogen, accompanied by ATP generation. Alcohol dehydrogenase $(\mathrm{ADH})$ is the key enzyme in the ethanol fermentation pathway and subsequently in the adaptive anaerobic metabolism of plant tissue [8]. It has been reported that the flooding tolerance of plants was proportional to the change in ADH activity in response to flooding. $A D H$ and $A D H$-like genes have been cloned from Arabidopsis thaliana, Zea mays, Cucumis sativus and Vitis vinifera [9-13]. ADH genes have been regarded as important candidates for genetic manipulation to achieve flooding tolerance in plants, through improving the adaptability to hypoxia $[14,15]$, although there have been relatively few studies on the response of $A D H$ genes to flooding in woody plants, such as T. hybrid 'Zhongshanshan 406'.

In the current study, we intend to identify and characterize the ThADHs of T. hybrid 'Zhongshanshan 406' and to study the expression and regulation of ThADHs in response to waterlogging stress, using real-time quantitative polymerase chain reaction (qPCR), to identify subcellular localization of the two alcohol dehydrogenases and to introduce the ThADHs into flooding-sensitive Populus by genetic transformation. The ultimate goal of our research is to reveal the molecular mechanism of waterlogging tolerance in $T$. hybrid 'Zhongshanshan 406'.

\section{Materials and Methods}

\subsection{Plant Materials and Waterlogging Treatment}

T. hybrid 'Zhongshanshan 406', which were planted, one per plant, in plastic pots containing 3:1:1 $(v / v / v)$ clay, vermiculite and perlite, were obtained from the Institute of Botany, Jiangsu Province and the Chinese Academy of Science, Nanjing, China $\left(35^{\circ} 50^{\prime} \mathrm{N}\right.$, $45^{\circ} 70^{\prime} \mathrm{E}$ ). The plants were irrigated fully every three days. Two-year-old $T$. hybrid 'Zhongshanshan $406^{\prime}$ with healthy and consistent growth were selected for stress treatment.

In early July, nine T. hybrid 'Zhongshanshan 406' plants of the similar size and development stage were selected for the experiment. The plant average height was $130 \pm 5 \mathrm{~cm}$ and the basal diameter was $9.6 \pm 0.15 \mathrm{~mm}$. Normal irrigation (CK, Supplementary PIC 1), half-flooding (HF, Supplementary PIC 2) and total-submergence (TS, Supplementary PIC 3) were set up as the three treatments. The CK plants were watered normally and placed outside but adjacent to the tanks. The HF plants were flooded with a water level depth as $20 \mathrm{~cm}$ above the soil surface. The TS plants were completely submerged in the water. Five replicate plants were used for each treatment. On the 0 , 10th, 20th, 30th, 40th, 50th and 60th days of the treatments, the leaf, stem and root tissues of CK, HF and TS plants were sampled and frozen in liquid nitrogen immediately and stored at $-80^{\circ} \mathrm{C}$ prior for RNA and DNA extraction.

The elite clone of hybrid poplars (Populus davidiana $\times$ P. bolleana), which were used for transient expression and genetic transformation studies, were cultivated on MS (Murashige and Skoog medium) agar medium supplemented with $0.3 \%(w / v)$ Gelrite and 3.0\% $(w / v)$ sucrose in a growth chamber at $25^{\circ} \mathrm{C}$ with a $16 / 8 \mathrm{~h}$ photoperiod. Protoplasts were isolated from the leaves of 42-day-old Populus and genetic transformation was carried out with 28-day-old Populus.

\subsection{Full-Length cDNA Cloning}

We extracted the total RNA from the different organs using the RNeasy ${ }^{\circledR}$ Plant Mini Kit (Qiagen, Dusseldorf, Germany). DNA contaminants were removed by DNase I (Takara, 
Dalian, China). Two ThADH genes sequence fragments were obtained from the transcriptome data from $T$. hybrid 'Zhongshanshan 406' plants exposed to waterlogging stress [7] and all primers were designed using the Oligo7 primer designer (http:/ / www.mbinsights.com/). Then, $3^{\prime}$ RACE (rapid amplification of cDNA ends) PCR and $5^{\prime}$ RACE PCR were performed according to the manufacturer's instructions (Takara, Dalian, China). The PCR fragments were ligated into the pMD T-19vector (Takara, Dalian, China) for sequencing. The sequences of the primers are shown in Table 1.

Table 1. Primer sequences of the genes for rapid amplification of cDNA ends (RACE), open reading frame (ORF) and real-time quantitative polymerase chain reaction (qPCR).

\begin{tabular}{|c|c|c|}
\hline Prime-ID & Forward PCR Primer $\left(5^{\prime}-3^{\prime}\right)$ & Reverse PCR Primer (5'-3') \\
\hline ThADH1_3OUTER & AAAGAAAGCATTACAGAGGCGTG & AAATCACTAGTGGAACGACGGTA \\
\hline ThADH1_3INNER & CAGTACCAGTACCAAATACCGAGGGTTGA & CCTATAGTGAAATCACTAGTGGAGGATCCGCG \\
\hline ThADH1_5OUTER & CTAATACGACTCACTATAGGGCAAGCAGTGGTAT & TTTTTCCTCCATTTGCTCGTTCTCAA \\
\hline ThADH1_5INNER & CTAATACGACTCACTATAGGGC & TCTGATTTCCGCAGCAAACTTTC \\
\hline ThADH1_ORF & ATGTCAAGCGCTACTGCAGG & ATCATCCAGTTTCATGACACATCT \\
\hline ThADH1_qRT-PCR & AGCGCTACTGCAGGGAAGGT & TTGATCCTGACTTCCATTGC \\
\hline ThADH4_3OUTER & TTCAAGAAGTTATAGCAGAGATGA & AAATCACTAGTGGAACGACGGTA \\
\hline ThADH4_3INNER & GGCCAAAACACAATTGCCTGGAATTGTGGAG & CCTATAGTGAAATCACTAGTGGAGGATCCGCG \\
\hline ThADH4_5OUTER & GAAACGTGCTTTCTTCСССТСССАT & GCTTTCTATTGTATTGGGCTTCGTCTT \\
\hline ThADH4_5INNER & GGATCCACCTGAACATCTTCTATTACCAGA & ACCAGACAAAGTTATTGGGAGCAGAGG \\
\hline ThADH4_ORF & ATGGAGATACAGAATGGAATA & GAAGTGAAGAACACATCTCA \\
\hline ThADH4_qRT-PCR & CAAAGTCCCTCTGTCT & AATATGCGAGGAAACGTG \\
\hline
\end{tabular}

\subsection{Bioinformatics and Statistical Analyses}

The online BLAST software was used to analyze the DNA and protein sequences (https:/ / blast.ncbi.nlm.nih.gov/Blast.cgi). The composition, physical and chemical characterization of the proteins were analyzed using the Expert Protein Analysis System (http://web.expasy.org/protparam/). Secondary structures of deduced amino acid sequences were predicted by the GOR IV secondary structure prediction method (https: / / npsa-prabi.ibcp.fr/cgi-bin/npsa_automat.pl?page=npsa_gor4.html). Protein subcellular localization prediction were performed using WoLF PSORT (https: / / www.genscript. com/psort/wolf_psort.html). Typical domain analysis was performed using the Pfam database (http:/ / pfam.sanger.ac.uk/). Full-length protein sequences of various species were obtained in the non-redundant $\mathrm{Nr}$ protein sequence repository of the public GenBank database, performed using BLAST. Multiple alignments of the two ADH proteins were performed with ClustalX (http:/ / www.clustal.org/clustal2/), using the default settings. Subsequently, a phylogenetic tree was constructed using the maximum likelihood method (1000 bootstraps), using MEGA7 software (http:/ / www.megasoftware.net/) [16].

\subsection{Quantitative Real-Time PCR Analysis}

To determine the expression profiles of ThADH1 and ThADH4, qPCR was performed on the Analitik Jena qTOWER2.2 PCR System (Biometra, Jena, Germany). The primers of the two ThADHs for qPCR were designed, based on their cDNA sequences. The adenine phosphoribosyltransferase (APRT, GenBank accession No. KX431853) gene was selected as the internal reference gene [17] (Table 1). Every sample was carried out in three biological independent replicates. The expression results were displayed in the form of the relative value $2^{-\Delta \Delta C t}$, where $\Delta C t$ represents the $C t$ value of the gene subtracted from that of the reference gene [18]. All data with three biological replicates were determined using oneway analysis of variance (ANOVA) and significant differences were calculated using the Duncan's multiple range tests (defined as $p<0.05$ ) by SPSS25.0 statistical analysis software. 


\subsection{Vector Construction}

The open reading frames (ORFs) of the ThADHs were amplified and subsequently ligated into the PCR8/GW/TOPO entry vector and then cloned into transient expression vector p2GWF7.0 and overexpression vector PH35GS, using the Gateway System (Invitrogen, Carlsbad, CA, USA). The genes were inserted downstream of the constitutive plant promoter CaMV35S in both vectors. The constructed vectors, 35S::ThADHs-GFP were introduced into Populus protoplasts for subcellular localizations of the ThADH proteins. The constructed vectors, 35S::ThADHs were transformed into Agrobacterium tumefaciens strain GV3101 for Populus transformation.

\subsection{Protoplast Transfection}

Hybrid poplar tissue culture seedlings (42-days-old) was grown in tissue culture and protoplasts were released, using cellulase and pectate lyase [19]. The binary vector p2GWF7.0, harboring the double cauliflower mosaic virus 35S (CaMV35S)-ADH-green fluorescent protein (GFP), was introduced into freshly obtained Populus protoplasts by polyethylene glycol (PEG) and then incubated for $20 \mathrm{~h}$ at $23^{\circ} \mathrm{C}$ before being observed and recorded. The GFP fluorescence signals were imaged using an OLYMPUS BX35 microscope (Olympus, Tokyo, Japan).

\subsection{Populus Transformation and Waterlogging Treatment}

The overexpressed binary vector PH35GS with ThADH1 and ThADH4 under the control of the CaMV35S promoter was transformed into Agrobacterium tumefaciens strain GV3101. The transformed A. tumefaciens strain was used to transform hybrid poplar as previously described [20]. After transformation, the non-transgenic plants and transgenic plants were grown on MS agar medium under 16/8 h of day/night at temperature of $25{ }^{\circ} \mathrm{C}$ for 30 days, Kanamycin was used to select for the transformation-positive plants and qPCR was performed to verify the putative transformants (Table 1). Before of the waterlogging treatment, 12 plants (4 non-transgenic Populus, 4 ThADH1-overexpressed transgenic Populus and 4 ThADH4-overexpressed transgenic Populus) were selected for the growth parameters analysis. The plant height was measured with ruler and then the plants were dried at $80^{\circ} \mathrm{C}$ until constant weight to derive their dry weights, ME104E electronic balance (METTLER, Zurich, Switzerland) was used to measure biomass. Data of growth parameters were analyzed with one-way analysis of variance (ANOVA) followed by Duncan's multiple range test at $p<0.05$ and the expression profiles of ThADH1 and ThADH4 in non-transgenic and transgenic Populus were detected by qPCR. Moreover, 8 non-transgenic Populus, 12 ThADH1-overexpressed transgenic Populus (line 1-3), 12 ThADH4-overexpressed transgenic Populus (line 1-3), a total of 32 plants were selected for waterlogging experiment with tissue culture bottles $(15 \mathrm{~cm} \times 8 \mathrm{~cm}$ each). After 20 days, the waterlogging stress was removed to obtain phenotype observations, measure the biomass and plant height.

\section{Results}

\subsection{Isolation and Characterization of ADH Genes from T. hybrid 'Zhongshanshan 406'}

Through the previous annotated transcriptome sequences, the full-length cDNAs of the two $A D H$ genes, namely ThADH1 and ThADH4, were cloned from the cDNA of T. hybrid 'Zhongshanshan 406', using the RACE technique. ThADH1 (Genbank ID: AWL83216) proved to be highly homologous to gymnosperms Pinus banksiana PbADH (83.06\%), whereas the homology of the amino acid sequence with that of A. thaliana AtADH1 was $76.86 \%$. The second T. hybrid 'Zhongshanshan 406' ADH gene, ThADH4 (Genbank ID:AWL83217), shared 71\% identity at the amino acid level with relict plant Amborella trichopoda AmADH4 (Genbank ID: XP 011620525.2). Sequence analysis demonstrated that the cDNAs of ThADH1 and ThADH4 encoded proteins of 381 and 403 amino acids (Supplementary Data 1), respectively, with predicted molecular weights (MW) of 41.03 and $43.7 \mathrm{kDa}$, as well as isoelectric points (pI) of 6.23 and 5.91, respectively. Protein subcellular 
localization predictions, using transient expression assays, indicated that the two ThADH proteins were both located in the cytoplasm, whereas ThADH4 protein was also located in the nucleus. The ThADH1 and ThADH4 proteins contained $15.22 \%$ and $16.13 \% \alpha$ helices $(\mathrm{Hh})$, respectively, a $32.55 \%$ and $29.53 \%$ of extended strands (Ee), respectively and $52.23 \%$ and $54.34 \%$ of random coils $(\mathrm{Cc})$, respectively. Interestingly, there was no zero $\beta$ turns (Tt) in ThADH1 and ThADH4 proteins (Table 2).

Table 2. Features of the ThADH1 and ThADH4 genes and their protein products.

\begin{tabular}{|c|c|c|c|c|c|c|c|c|c|c|c|}
\hline \multirow{2}{*}{ Gene_ID } & \multirow{2}{*}{$\begin{array}{l}\text { Full-Length } \\
\text { cDNA (bp) }\end{array}$} & \multirow{2}{*}{$5^{\prime}$-UTR (bp) } & \multirow{2}{*}{$3^{\prime}$-UTR (bp) } & \multirow{2}{*}{ ORF (bp) } & \multicolumn{3}{|c|}{ Predicted Peptide } & \multicolumn{4}{|c|}{ Secondary Structure Prediction } \\
\hline & & & & & MW (kDa) & pI & GRAVY & Hh (\%) & Ee $(\%)$ & $\operatorname{Tt}(\%)$ & Cc $(\%)$ \\
\hline ThADH1 & 1518 & 89 & 283 & 1146 & 41.03 & 6.23 & -0.024 & 15.22 & 32.55 & 0.00 & 52.23 \\
\hline ThADH4 & 1506 & 117 & 177 & 1212 & 43.7 & 5.91 & 0.064 & 16.13 & 29.53 & 0.00 & 54.34 \\
\hline
\end{tabular}

UTR: untranslated region; ORF: open reading frame; MW: molecular weight; pI: isoelectric point; GRAVY: grand average of hydropathicity; Hh: $\alpha$ helices; Ee: extended strands; Tt: $\beta$ turns; Cc: random coils.

\subsection{Multiple Alignment and Phylogenetic Analysis of ThADH1 and ThADH4}

Multiple alignments were carried out to show the identity of the protein sequences between ThADH1, ThADH4 and other known ADH proteins AtADH1, Quercus suber QsADH1 (GenBank ID: XP_023885013.1), AmADH4 and QsADH4 (GenBank ID: XP_023893720.1). The result showed that both ThADH proteins contained a large ADH_III_F_hyde domain, indicating that ThADH1 and ThADH4 were members of the plant-specific class III alcohol dehydrogenase protein family, with a conserved $\mathrm{ADH}_{-} \mathrm{N}$ domain located in the $\mathrm{N}$-terminal and an ADH_ZIN_N domain located in the C-terminal of the ThADH1 and ThADH4 proteins. Although the amino acid sequences exhibited slight differences, the genes exhibited high similarities with one another in the ADH domain (Figure 1).

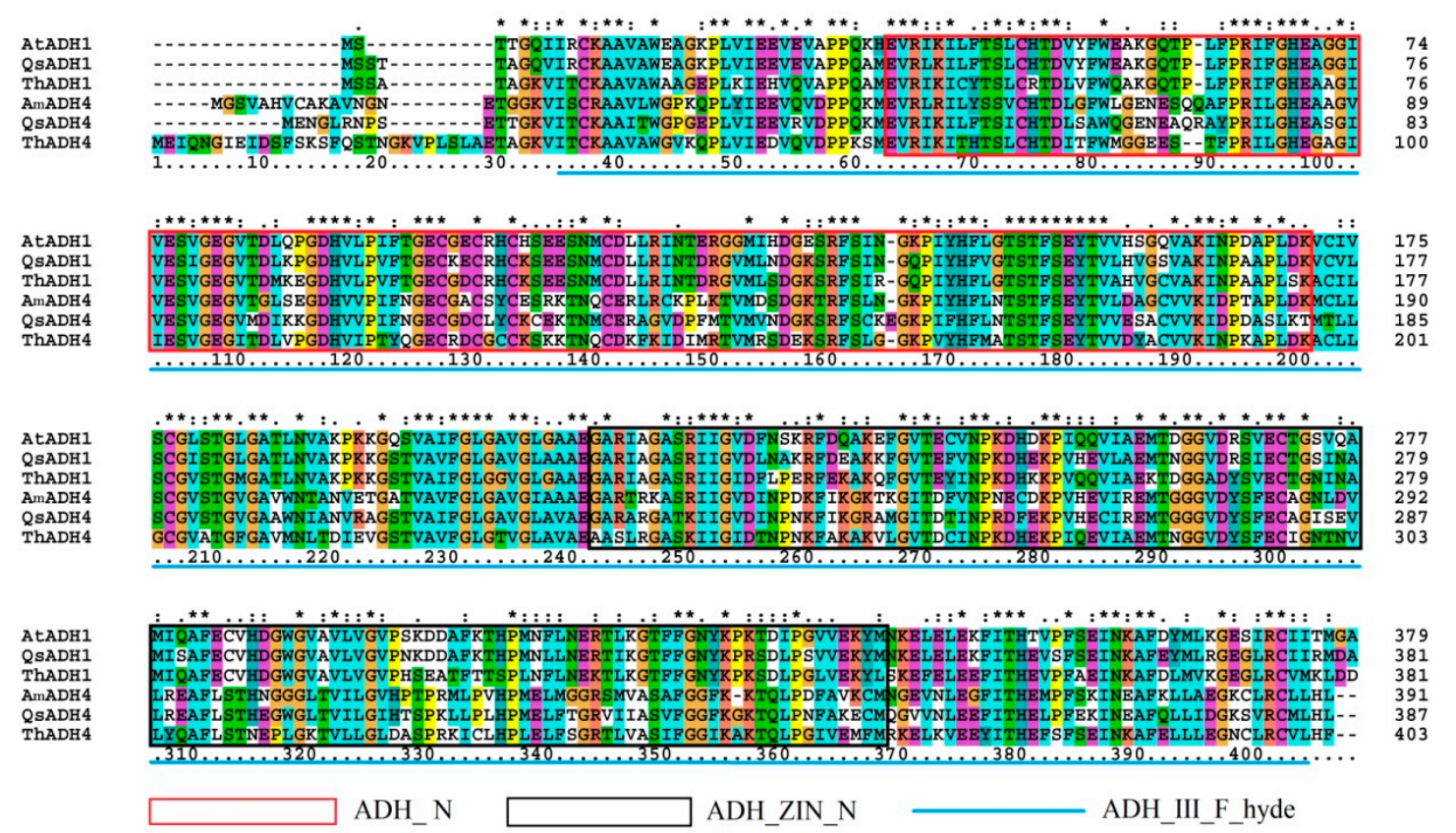

Figure 1. Alignment of the amino acid sequences of ThADH1, ThADH4 with Arabidopsis thaliana AtADH1, Quercus suber QsADH1, QsADH4 and Amborella trichopoda AmADH4. The highly conserved regions of the ADH proteins are shown by the red, black boxes and blue line, which represent the following conserved domains: ADH_N domain, ADH_ZIN_N domain and ADH_III_F_hyde domain.

To further understand the evolutionary relationships between the ThADH proteins and the ADH proteins of other species. The amino acid sequences of ThADH1, ThADH4 and $35 \mathrm{ADH}$ proteins from other species were used to construct a phylogenetic tree by MEGA7 software. Phylogenetic analysis, based on multiple sequence alignments, revealed 
that all these proteins could be divided into two distinct groups: ThADH1, closely related to AtADH1 and other ADH1 proteins, was positioned in the ADH1 clade, whereas ThADH4 belonged to the other group, including AmADH4, Phoenix dactylifera PdADH4 (GenBank ID: XP008811874.1), Sesamum indicum SiADH4 (GenBank ID: XP011070396.1) and so on, were located in the ADH4 clade (Figure 2).

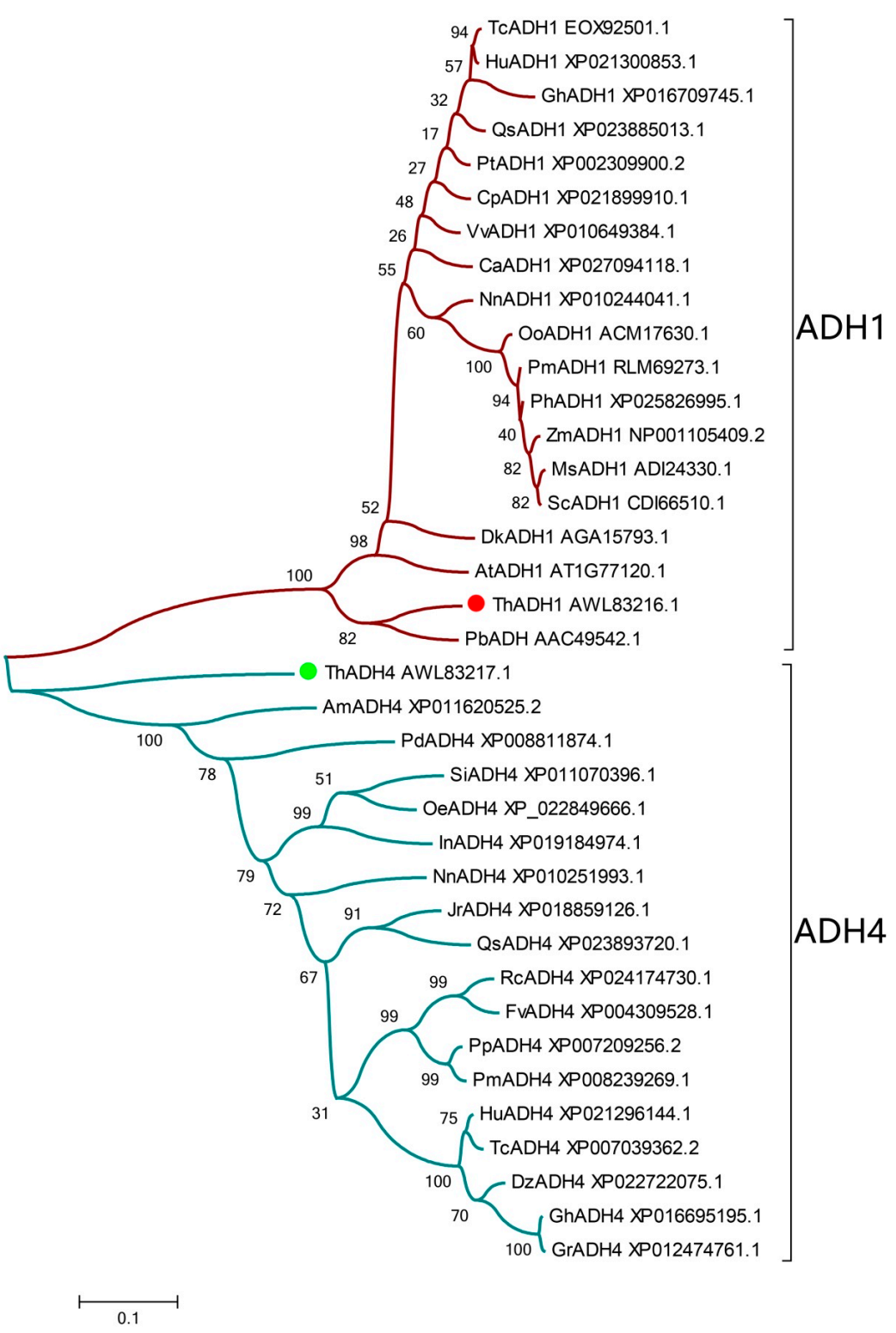

Figure 2. Phylogenetic analysis of the proteins of the $A D H$ gene family. The phylogenetic tree was constructed using MEGA 7.0 with the maximum likelihood method using 1000 replicate bootstrap tests. Numbers near to the nodes indicate bootstrap values obtained from 1000 replications. The 35 proteins were clustered into two distinct groups: ADH1 and ADH4, as indicated. At, Arabidopsis thaliana; Os, Oryza sativa; $\mathrm{Pb}$, Pinus banksiana; $\mathrm{Pt}$, Populus trichocarpa; Hu, Herrania umbratica; Tc, Theobroma cacao; Vv, Vitis vinifera; Pa, Prunus avium; Qs, Quercus suber; Pm, Panicum miliaceum; Zm, Zea mays; Dl, Dimocarpus longan; Rc, Rosa chinensis; Gh, Gossypium hirsutum; Ca, Coffea arabica; Sh, Saccharum hybrid cultivar; Ms, Miscanthus sinensis; Nn, Nelumbo nucifera; Dk, Diospyros kaki; Oo, Oryza officinalis; Am, Amborella trichopoda; Nn, Nelumbo nucifera; Dz, Durio zibethinus; Gr, Gossypium raimondii; Rc, Rosa chinensis; Jr, Juglans regia; Oe, Olea europaea var. sylvestris; Pm, Prunus mume; $\mathrm{Pp}$, Prunus persica; Pd, Phoenix dactylifera; In, Ipomoea nil; Se, Sesamum indicum; Fv, Fragaria vesca subsp. vesca; Zm, Zea mays; $\mathrm{Cp}$, Carica papaya; $\mathrm{Ph}$, Panicum hallii; $\mathrm{Sc}$, Saccharum hybrid cultivar; $\mathrm{Si}$, Sesamum indicum. 


\subsection{Expression Patterns of ThADH1 and ThADH4 Genes}

To analyze the expression of ThADH1 and ThADH4, the two ThADH genes were expressed in the three main organs (stems, leaves and roots) of entire T. hybrid 'Zhongshanshan 406' plants at three levels of treatments and seven time points, analyzed by qPCR (Supplementary Data 2). The mRNA expression patterns in response to waterlogging were similar, with an apparent increase-decrease trend. Gene expression in roots was much higher than that in stems and leaves under half-flooding (HF), total submergence (TS) and non-flooding (CK) conditions. The expression levels of the two genes exhibited a continuous rise in expression from 0day to 50day of the TS and HF treatments, in both roots or stems, the greatest accumulation of both ThADH1 and ThADH4 transcripts was observed on the 50th day, from which point gene expression began to decline (Figure 3).
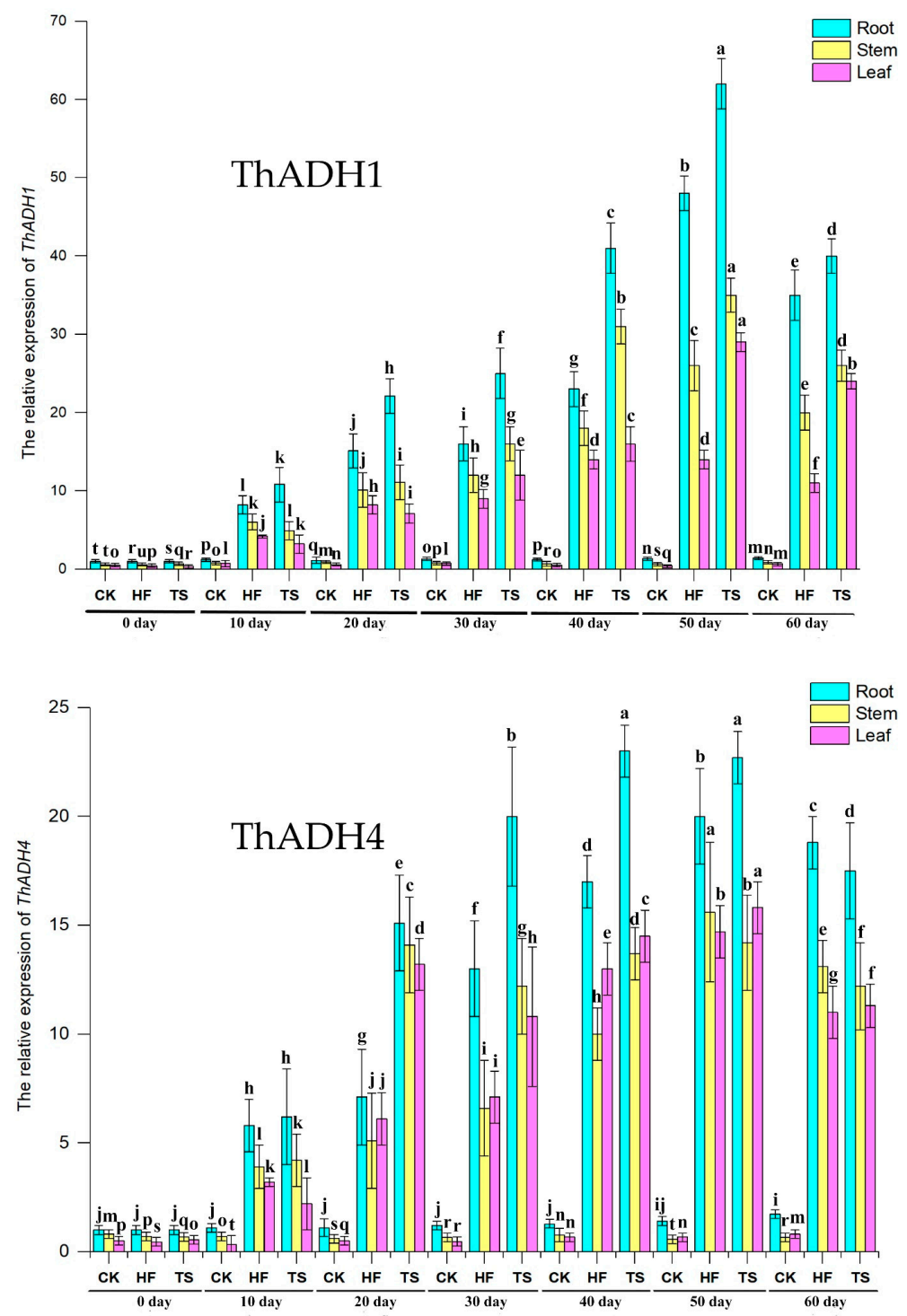

Figure 3. Temporal expression patterns of ThADH1 and ThADH4 by qPCR. CK, HF and TS represent non-flooding, half-flooding and total submergence. The gene expression level in the root at 0 day was set to 1. ThADH1 and ThADH4 genes expression in root, stem and leaf of T. hybrid 'Zhongshanshan 406 'at different times (from 0 day to 60 day). For qPCR, the APRT gene was used as the internal control and the relative transcript levels were calculated using the comparative delta-Ct method. All the qPCR data are shown as the mean \pm standard deviation (error bar) of three biological replicates. Means with different letters are significantly different at $p<0.05$ as determined by one-way ANOVA with Duncan's multiple range tests. The same below. 


\subsection{Subcellular Localization of ThADH1 and ThADH4 Proteins}

In order to probe the subcellular localizations of the ThADH proteins, under the control of $35 \mathrm{~S}$ CaMV promoter, the GFP was fused to the C-terminus of the ADH proteins and the chimeral genes introduced into hybrid poplar (Populus davidiana $\times$ Populus bolleana) protoplasts for expression in a transient gene expression assay. As a positive control, we detected 35S::GFP fusion protein signals in the nucleus, cytoplasm and cytomembrane of Populus protoplasts and the fluorescent signal of ThADH1 was detected within the nucleus and the cytoplasm, whereas no nuclear ThADH4 protein fluorescent signal could be found in the nucleus, which was located only in the cytoplasm (Figure 4). These results implied that ThADH1 and ThADH4 may have different biological functions in plants.
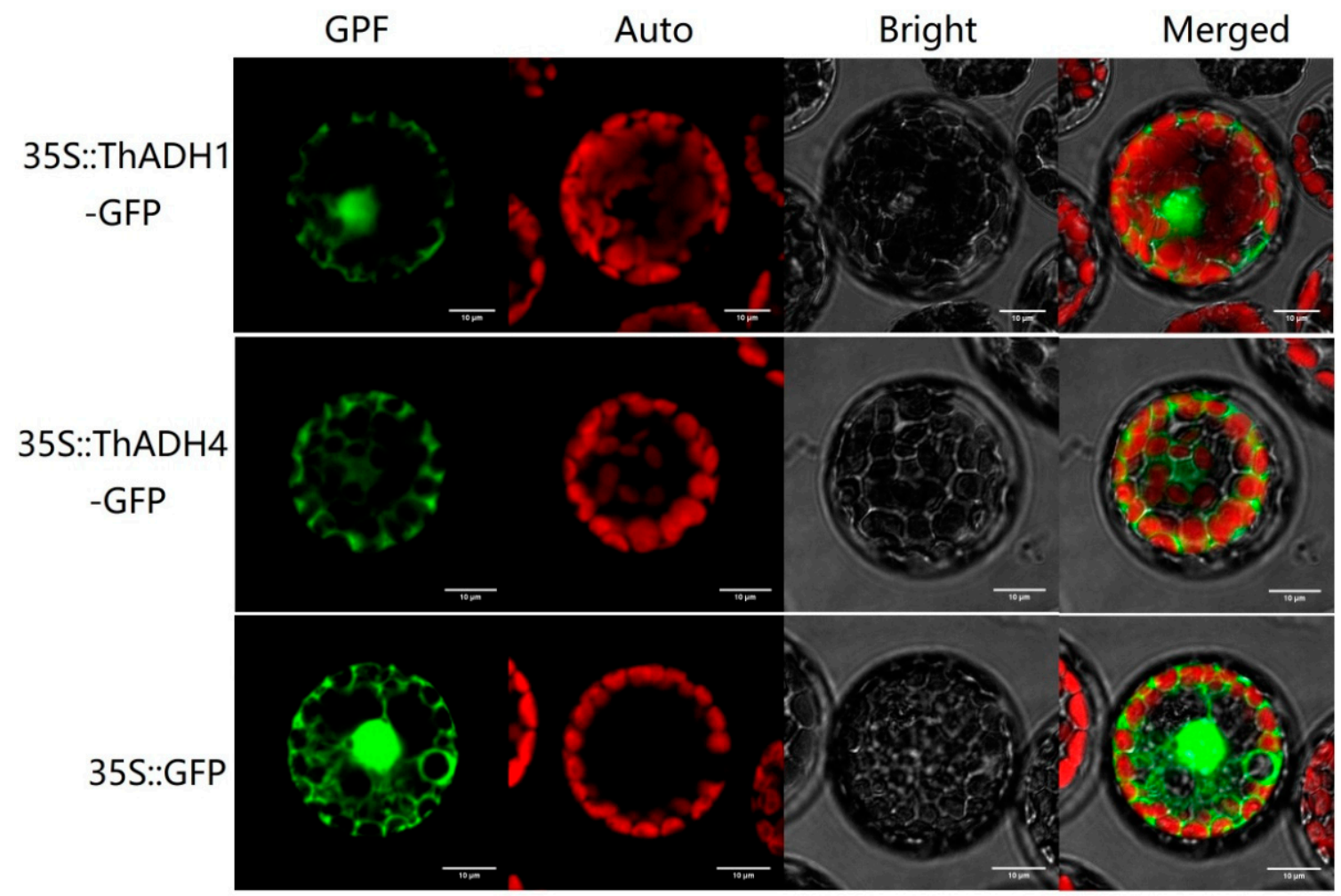

Figure 4. Subcellular localization of ThADH1 and ThADH4 in poplar protoplasts. Green fluorescent protein (GFP), chlorophyll autofluorescence (Auto), bright and merged images are shown. Scale bar $=10 \mu \mathrm{m}$. The 35::GFP fusion protein was used as the positive protein control.

3.5. Heterologous Overexpression of ThADH 1 and ThADH 4 in Populus and Comparison of Waterlogging Tolerance in Non-Transgenic and Transgenic Populus

To reveal the biological functions of ThADH1 and ThADH4, we overexpressed ThADH1 and ThADH4 in Populus. Phenotypic observation demonstrated that the growth rates of the ThADH1- and ThADH4-overexpressed transgenic plants were significantly different from that of non-transgenic plants. After 30 days of growth, overexpression of the ThADH1 accelerated the growth rate of the transgenic Populus but the growth rate of ThADH4overexpressed transgenic Populus was lower than that of the control (Figure 5A,C). In particular, compared with non-transgenic Populus, the biomass and plant height of ThADH1overexpressed transgenic Populus were higher by $15.6 \%$ and $49.4 \%$, respectively (Table 3 ). Meanwhile, the petioles and stems of the ThADH4-overexpressed transgenic Populus were significantly shorter than that of non-transgenic and ThADH1 transgenic Populus, resulting in a dwarfed phenotype. For this reason, the biomass and plant height of non-transgenic Populus was $50.4 \%$ and $51.2 \%$ higher than that of the ThADH4 transgenic Populus (Table 3). The expression level of the ThADH1 gene in the transgenic Populus ThADH1 Lines 1-3 was 
531-580 times higher than that in non-transgenic Populus, whereas the expression level of the ThADH4 gene in the transgenic Populus ThADH4 Lines 1-3 was 1331-1950 times higher than that in the non-transgenic Populus. Interestingly, ThADH4 gene was detected in ThADH1-overexpressed transgenic Populus and ThADH1 gene was also detected in ThADH4-overexpressed transgenic Populus, although their expression levels were relatively low (Supplementary PIC 4).
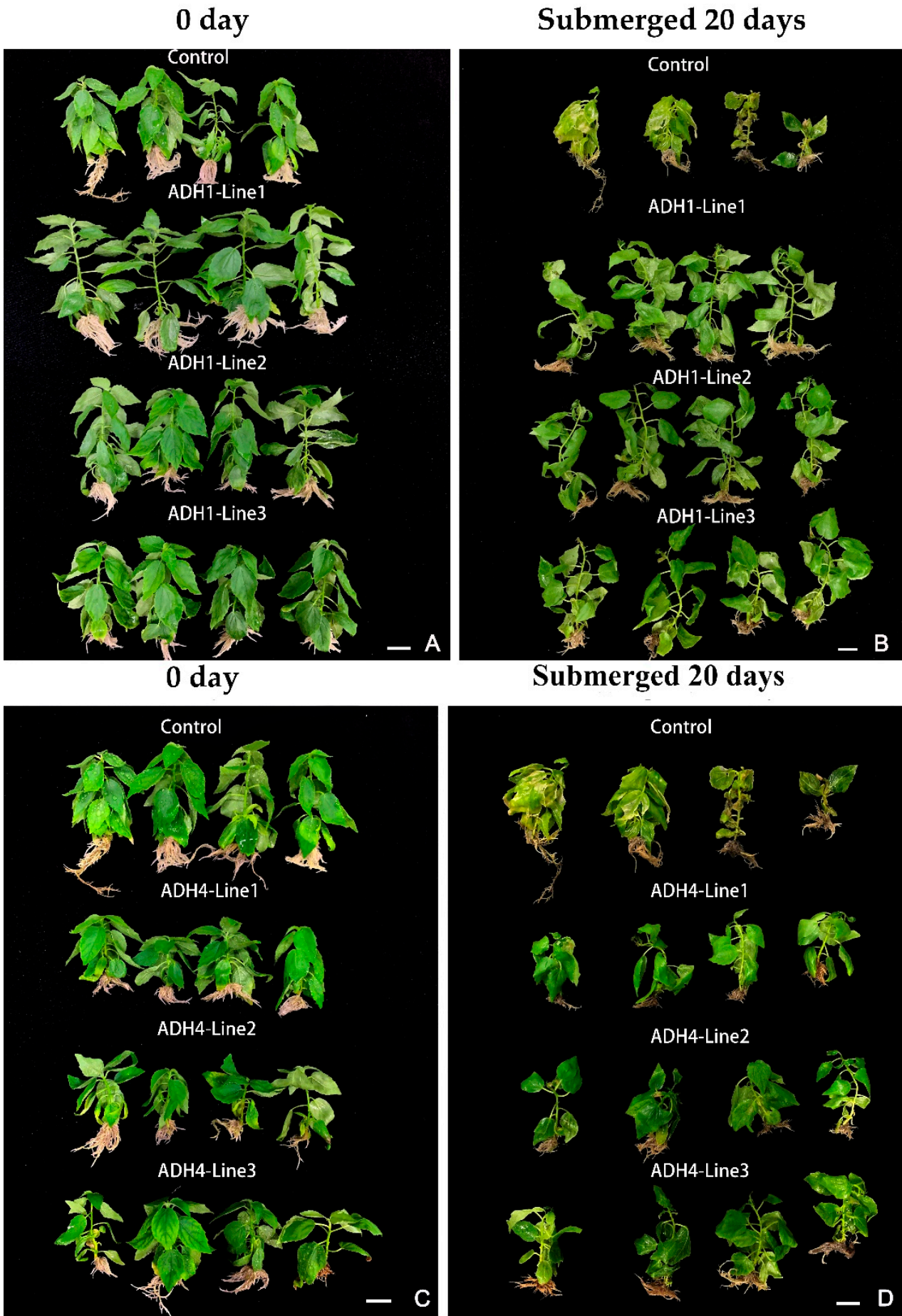

Figure 5. Overexpression of ThADH1 or ThADH4 conferred enhanced waterlogging tolerance in plants. (A,C) Phenotypic changes of transgenic Populus after 30 days of growth ADH1/ADH4 Lines 1-3 and non-transgenic (Control), respectively. (B,D) 30 days WT and transgenic Populus were photographed at the conclusion of submersion in deionized water for an additional 20 days. Bar scale $=1.0 \mathrm{~cm}$. 
Table 3. Growth parameters of transgenic Populus and Non-transgenic Populus at $0 \mathrm{~d}$ and $20 \mathrm{~d}$ of flooding stress.

\begin{tabular}{cccc}
\hline & & Biomass (g) & Height (cm) \\
\hline \multirow{2}{*}{ 0 Day } & ThADH1-Transgenic Populus & $0.5449 \pm 0.015 \mathrm{~b}$ & $11.5 \pm 0.08 \mathrm{~b}$ \\
& ThADH4-Transgenic Populus & $0.3133 \pm 0.009 \mathrm{f}$ & $5.275 \pm 0.09 \mathrm{e}$ \\
& Non-transgenic Populus & $0.4713 \pm 0.004 \mathrm{c}$ & $7.975 \pm 0.17 \mathrm{c}$ \\
\hline \multirow{3}{*}{ Submerged 20 Days } & ThADH1-Transgenic Populus & $0.6161 \pm 0.011 \mathrm{a}$ & $12.025 \pm 0.22 \mathrm{a}$ \\
& ThADH4-Transgenic Populus & $0.3471 \pm 0.014 \mathrm{e}$ & $6.075 \pm 0.09 \mathrm{~d}$ \\
& Non-transgenic Populus & $0.4131 \pm 0.004 \mathrm{~d}$ & $8.05 \pm 0.13 \mathrm{c}$ \\
\hline
\end{tabular}

Data presented are means \pm standard errors $(n=4)$. Means followed by different letters in same column are significantly different according to Duncan's Multiple Range Test at $5 \%$ level.

In order to further explore the waterlogging tolerance of ThADH1 and ThADH4, we completely flooded 30-day-old ThADH1- and ThADH4-overexpressed transgenic plants for 20 days and the non-transgenic Populus was used as a control (Supplementary PIC5). Compared with ThADH1- and ThADH4-overexpressed transgenic Populus, the morphology of the non-transgenic Populus has changed significantly and its leaves and segments appeared to turn yellow or transparent, even rotted (Supplementary PIC6). However, the growth of ThADH1- and ThADH4-overexpressed transgenic plants was slightly affected at the terminal buds (Figure 5B,D) and some ThADH1 transgenic Populus grew new adventitious roots under waterlogging stress (Supplementary PIC7). Furthermore, compared with non-flooding (at 0 day of flooding stress) plants, the biomass and plant height of ThADH1- and ThADH4-overexpressed transgenic Populus were significantly enhanced (13\% and $4.6 \%, 10.8 \%$ and $15.2 \%$, respectively) after submerged treatment for 20 days. However, non-elongating height and decreased biomass of non-transgenic plants were observed (Table. 3). These results implied that overexpression of ThADH1 and ThADH4 conferred tolerance to submergence and hypoxia stresses, albeit via different pathways and that they were the positive regulatory factors of waterlogging tolerance of $T$. hybrid 'Zhongshanshan 406'.

\section{Discussion}

The plant $A D H$ gene family is a small polygenic family, $A$. thaliana has only one $A D H$ gene [21], whereas rice (Oryza sativa) and maize (Z. mays) have three copies of $A D H$ genes each [22], whereas soybean (Glycine max) has six copies of $A D H$ genes, five of which are induced to express under waterlogging stress [23]. At present, the study of $A D H s$ is mainly concentrated on herbaceous plants, with few studies in woody plants. In this study, we described the identification and characterization of two T. hybrid 'Zhongshanshan 406' $A D H$ genes, namely ThADH1 and ThADH4. According to the results of National Center for Biotechnology Information (NCBI) homology alignment, ThADH1 and ThADH4 showed high similarities with respect to the amino acid sequences of ADH proteins from other plants. ThADH1 shared $76.86 \%$ identity at the amino acid level with $A$. thaliana AtADH1, while ThADH4 shared $71 \%$ identity at the amino acid level with Amborella trichopoda AmADH4, indicating that ThADH1 and ThADH4 are relatively highly conserved and this conservatism may be related to some specific functions. ThADHs are $\mathrm{Zn}$-binding enzymes with two conserved domains: one ADH_N and one ADH_zinc_N domain. Both ThADHs have a large conserved DNA-binding domain (adh_III_F_hyde domain); almost all plant $\mathrm{ADH}$ proteins have these conserved domains, which may explain the reason for the highly conserved nature of plant alcohol dehydrogenases [24,25].

Recent studies have shown that most ADH proteins are localized in the cytoplasm. In upland cotton (Gossypium hirsutum), all 28 GhADHs were shown to be located in the cytoplasm, with some of the proteins also being located on the pericytoplasmic or extracellular membrane [26]. In this study, subcellular localization investigations showed that the ThADH4 protein was located only in the cytoplasm, whereas the ThADH1 protein was located not only in the cytoplasm but also in the nucleus. This result was consistent 
with our previous WoLF PSORT software predictions and may be related to the functions of the proteins and the biological processes involved. In Arabidopsis, hypoxia stress-related transcription factor RAP2.12 can specifically bind to ADH1 in the nucleus [27]. Therefore, we speculate that whether ThADH1 protein is located in the nucleus is related to the function of upstream transcription factors, which needs further study.

Under anaerobic conditions, alcoholic fermentation in higher plants is essential for supplying $\mathrm{NAD}^{+}$to the glycolytic pathway, which is in charge of ATP synthesis and $A D H$ genes are considered to play a key role in hypoxic stress response [28]. A total of $22 \mathrm{TaADH}$ genes were identified from the wheat genome, three of which, namely TaADH1/2, TaADH3 and TaADH9, played an important role in coping with waterlogging stress and which can be used as an effective basis on which to screen for waterlogging-tolerant wheat varieties [29]. Under conditions of hypoxia, the transcription levels of $\mathrm{ZmADH1}$ and $\mathrm{ZmADH} 2$ in maize both increased rapidly and then decreased under anaerobic conditions [30]. In the current study, to further investigate the two isolated ThADH genes, we focused on their expression patterns under long-term waterlogging stress. The extent of up-regulation of ThADH1 and ThADH4 under waterlogging stress was consistent in the three organs tested, indicating that, although hypoxic stress was perceived by roots, the signal arrived shortly at the stems and leaves, inducing the expression of $A D H$ genes in those organs. When plants were completely submerged in water, the expression of ThADH1 and ThADH4 were increased and the elevated expression pattern were continued until 50th day, when the expression levels of ThADH1 and ThADH4 in the roots reached to 63 and 23 times of 0 day, respectively. Research on rice and Coix lacryma-jobi, both grasses, have shown that both the OsADH1 and $\mathrm{ClADH} 1$ genes were sensitive to flooding stress, with the expression of these genes reaching the highest levels within six hours of short-term flood stress [31,32]. Results showed that there were some differences in expression response patterns of $A D H$ genes between different plants under long-term and short-term waterlogging stress and that ThADH1 and ThADH4 could also be used as a marker of waterlogging stress. The sequences of ThADH1 and ThADH4 were similar to each other in terms of the ORF sequences; gene expression patterns also showed similar trends, although ThADH1 was up-regulated to a much higher level than was ThADH4 under waterlogging stress treatment. This result showed that, of the two genes, ThADH1 may play the more important role in the response of Taxodium to hypoxic stress.

$A D H$ is one of the most important candidate genes for genetic manipulation to provide waterlogging tolerance to plants via increasing adaptability to hypoxic responses. Maize and Arabidopsis ADH-null mutants showed lower tolerance to anaerobic conditions [33-35]. In rice, the inhibition of $A D H$ resulted in a decrease in seed vigor under anoxic conditions, whereas overexpression of $A D H$ promoted plant growth under waterlogging conditions [31,36]. However, the role of $A D H$ during waterlogging-tolerance signaling has not been established in coniferous species. In the present study, we tested whether transgenic Populus carrying ThADHs exhibited increased waterlogging tolerance. Therefore, we overexpressed the two ThADH genes in hybrid poplars to identify their functions. Interestingly, transgenic plants ThADH1 Lines 1-3 and ThADH4 Lines 1-3 showed completely opposite phenotypes. Saika [18] demonstrated that a point mutation in the OsADH1 gene is associated with inhibition of coleoptile elongation in rice under submergence conditions, indicating that $A D H$ genes may affected the growth of plants. Sometimes, the dwarfing phenotype of plant may have a negative impact on the plant's waterlogging tolerance but waterlogging experiments showed that overexpression of ThADH1 or ThADH4 genes enhanced the withstand flooding resistance of Populus (Supplementary Data 3). Compared with the control, the phenotype of ThADH1- or ThADH4-transgenic Populus was closer to the non-flooding state and they can grow normally under flooding stress. These results inferred that ThADH1 and ThADH4, as positive regulators, improve tolerance to waterlogging or hypoxia stresses of $T$. hybrid 'Zhongshanshan 406' through different signal pathways. 
Taken together, these results strongly indicated that each of ThADH1 and ThADH4 plays an essential role in tolerance to waterlogging stress in $T$. hybrid 'Zhongshanshan 406 '. Further study is necessary to explore the response mechanism of ThADH1 and ThADH4 to waterlogging stress, to identify the association between them and other genes, such as $P D C$ (Pyruvate decarboxylase) and ALDH (Acetaldehyde dehydrogenasein) the glycolysis pathway, to find their upstream and downstream genes and to explore whether the two genes function singly or together.

\section{Conclusions}

In this study, we isolated and characterized two ThADHs genes from T. hybrid 'Zhongshanshan 406'. The expression levels of the two genes in roots, stems and leaves exhibited trends of continuous rise from 0 to 50 day under the half-flooding and total submergence treatments. Furthermore, we demonstrated that overexpression of ThADH1 and ThADH4 in Populus could significantly increase waterlogging tolerance of plant, inferring that ThADH1 and ThADH4 may play essential parts in tolerance to waterlogging stress in T. hybrid 'Zhongshanshan 406 '. Altogether, our findings will be beneficial to enrich the understanding of molecular mechanism of flooding tolerance in $T$. 'Zhongshanshan' and provide a theoretical basis for the germplasm innovation of Taxodium.

Supplementary Materials: The following are available online at https: / /www.mdpi.com/2073-442 5/12/2/225/s1, Supplementary Data 1: The full-length cDNA and amino acid sequences of ThADH1 and ThADH4. Supplementary Data 2: Gene expression of ThADH1 and ThADH4. Supplementary Data 3: Growth parameters of transgenic Populus and Non-transgenic Populus at 0d and 20d of flooding. Supplementary PIC 1-3. Normal irrigation (CK), half-flooding (HF) and total submergence (TS) of T. hybrid 'Zhongshanshan 406'. Supplementary PIC 4: Histogram of gene expression in transgenic plants. Supplementary PIC 5: The 30-day-old non-transgenic plants, ThADH1- and ThADH4-overexpressed transgenic plants were completely flooded in tissue culture bottles. Supplementary PIC 6: Phenotypic changes of leaves and stem segments of some non-transgenic plants and transgenic plants after submerged 20 days. Supplementary PIC 7: The enlarged images of nontransgenic and ThADH1-overexpressed transgenic plants after 20 days of waterlogging treatment.

Author Contributions: J.H. and L.X. conceived and designed the project. L.X. performed the experiments, wrote the manuscript and analyzed the data, with contributions from Y.Y. (Ying Yang), Z.W., F.Z. and Y.Y. (Yunlong Yin). X.P. helped to complete the experiments. D.L.C. revised the manuscript. All authors have read and agreed to the published version of the manuscript.

Funding: This study was supported by the National Natural Science Foundation of China (31870592, 31971660). Independent innovation research projects of Institute of Botany, Jiangsu Province and Chinese Academy of Sciences (JSPKLB202048), Jiangsu province policy guidance program—special project for introducing foreign talents (BX2020010).

Institutional Review Board Statement: The study did not require ethical approval, for studies not involving humans or animals.

Informed Consent Statement: Studies not involving humans.

Conflicts of Interest: The authors declare no conflict of interest.

\section{References}

1. Chang, C.; Meyerowitz, E.M. Molecular cloning and DNA sequence of the Arabidopsis thaliana alcohol dehydrogenase gene. Proc. Natl. Acad. Sci. USA 1986, 83, 1408-1412. [CrossRef]

2. Dennis, E.S.; Gerlach, W.L.; Pryor, A.J.; Bennetzen, J.L.; Inglis, A.; Llewllyn, D.; Sachs, M.M.; Ferl, R.J.; Peacock, W.J. Molecular analysis of the alcohol dehydrogenase (Adh1) gene of maize. Nucl. Acids Res. 1984, 12, 3983-4000. [CrossRef] [PubMed]

3. Xie, Y.; $\mathrm{Wu}, \mathrm{R}$. Rice alcohol dehydrogenase genes: Anaerobic induction, organ specific expression and characterization of cDNA clones. Plant Mol. Biol. 1989, 13, 53-68. [CrossRef] [PubMed]

4. Torregrosa, L.; Pradal, M.; Souquet, J.M.; Rambert, M.; Gunata, Z.; Tesniere, C. Manipulation of $V v A d h$ to investigate its function in grape berry development. Plant Sci. 2008, 174, 149-155. [CrossRef]

5. Xuewen, X.; Huihui, W.; Xiaohua, Q.; Qiang, X.; Xuehao, C. Waterlogging-induced increase in fermentation and related gene expression in the root of cucumber (Cucumis sativus L.). Sci. Hortic. 2014, 179, 388-395. [CrossRef] 
6. Kennedy, R.A.; Fox, R.T. Anaerobic Metabolism in Plants. Plant Physiol. 1992, 100, 1-6. [CrossRef]

7. Bailey-Serres, J.; Voesenek, L.A.C.J. Flooding stress: Acclimations and genetic diversity. Annu. Rev. Plant Biol. 2008, 59, 313-318. [CrossRef]

8. Bhupesh, T.; Mande, S.C. Conserved structural features and sequence patterns in the GroES fold family. Protein Eng. 1999, 12, 815-818.

9. Zhang, J.Y.; Wang, G.; Huang, S.N.; Xuan, J.P.; Guo, Z.R. Functions of Alcohol Dehydrogenase Family in Abiotic Stress Responses in Plants. Chin. Agric. Sci. Bull. 2015, 31, 246-250.

10. Ellis, M.H.; Setter, T.L. Hypoxia induces anoxia tolerance in completely submerged rice seedlings. J. Plant Physiol. 1999, 154, 219-230. [CrossRef]

11. Chen, D.Q.; Zhu, Y.F.; Wu, G.Q.; Li, Y.N. Characterization Analysis of Response of Alcohol Dehydrogenase Gene (ADH1) in Coix lacroyma> /em> jobi L. to Waterlogging Stress. Adv. J. Food Sci. Technol. 2012, 4, 417-425.

12. Dolferus, R.; Marbaix, G.; Jacobs, M. Alcohol dehydrogenase in Arabidopsis: Analysis of the induction phenomenon in plantlets and tissue cultures. Mol. Gen. Genet. MGG 1985, 199, 256-264. [CrossRef]

13. Matsumura, H.; Takano, T.; Takeda, G.; Uchimiya, H. Adh1 is transcriptionally active but its translational product is reduced in a rad mutant of rice (Oryza sativa L.), which is vulnerable to submergence stress. Theor. Appl. Genet. 1998, 97, 1197-1203. [CrossRef]

14. Jacobs, M.; Dolferus, R.; Bossche, D. Isolation and biochemical analysis of ethyl methanesulfonate-induced alcohol dehydrogenase null mutants of Arabidopsis thaliana (L.) Heynh. Biochem. Genet. 1988, 26, 105-122. [CrossRef]

15. Hirokazu, T.; Hank, G.; Hideo, M.; Nobuhiro, T.; Mikio, N. Rice alcohol dehydrogenase 1 promotes survival and has a major impact on carbohydrate metabolism in the embryo and endosperm when seeds are germinated in partially oxygenated water. Ann. Bot. 2014, 113, 851-859.

16. Jiang, M.; Deng, H.; Cai, Q.; Wu, G. Species richness in a riparian plant community along the banks of the Xiangxi River, the Three Gorges region. Int. J. Sustain. Dev. World Ecol. 2005, 12, 60-67. [CrossRef]

17. Wang, P.; Zhang, Q.; Xu, Y.S.; Yu, F.H. Effects of water level fluctuation on the growth of submerged macrophyte communities. Flora-Morphol. Distrib. Funct. Ecol. Plants 2016, 223, 83-89. [CrossRef]

18. Yu, X.; Luo, N.; Yan, J.; Tang, J.; Liu, S.; Jiang, Y. Differential growth response and carbohydrate metabolism of global collection of perennial ryegrass accessions to submergence and recovery following de-submergence. J. Plant Physiol. 2012, 169, 1040-1049. [CrossRef]

19. Wang, Z.Q.; Yin, Y.L.; Hua, J.F.; Fan, W.C.; Xuan, L. Cloning and Characterization of ThSHRs and ThSCR Transcription Factors in Taxodium Hybrid 'Zhongshanshan 406'. Genes 2017, 8, 185. [CrossRef]

20. Yin, Y.L.; Yu, C.G.; Hua, J.F.; Huan, J.J.; Han, L.W.; Qi, B.Y.; Ren, P.; Wu, X.H.; Qi, X.C. A trial on the silviculture of Taxodium hybrid 'Zhonshanshan118' planted in the hydro-fluctuation belt of the Three Gorges Reservoir within the Wanzhou district area of Chongqing City. China For. Sci. Technol. 2014, 2, 110-114.

21. Fan, W.; Yang, Y.; Wang, Z.; Yin, Y.; Yu, C.; Shi, Q.; Guo, J.; Xuan, L.; Hua, J. Molecular cloning and expression analysis of three ThERFs involved in the response to waterlogging stress of Taxodium 'Zhongshanshan406' and subcellular localization of the gene products. Peer] 2018, 6, 1-17. [CrossRef] [PubMed]

22. Qi, B.; Yang, Y.; Yin, Y.L.; Xu, M.; Li, H.G. De novo sequencing, assembly and analysis of the Taxodium 'Zhongshansa' roots and shoots transcriptome in response to short-term waterlogging. BMC Plant Biol. 2014, 14, 201-218. [CrossRef] [PubMed]

23. Tadege, M.; Dupuis, I.I.; Kuhlemeier, C. Ethanolic fermentation: New functions for an old pathway. Trends Plant Sci. 1999, 4, 320-325. [CrossRef]

24. Kumar, S.; Stecher, G.; Tamura, K. MEGA7: Molecular evolutionary genetics analysis version 7.0 for bigger datasets. Mol. Biol. Evol. 2016, 33, 1870-1874. [CrossRef] [PubMed]

25. Wang, Z.; Gu, C.; Xuan, L.; Hua, J.; Shi, Q.; Fan, W.; Yin, Y.; Yu, F. Identification of suitable reference genes in Taxodium 'Zhongshanshan' under abiotic stresses. Trees 2017, 31, 1519-1530. [CrossRef]

26. Schmittgen, T.D.; Livak, K.J. Analyzing real-time PCR data by the comparative CT method. Nat. Protoc. 2008, 3, 1101-1108. [CrossRef] [PubMed]

27. Tan, B.; Xu, M.; Chen, Y.; Huang, M. Transient expression for functional gene analysis using Populus protoplasts. Plant Cell Tissue Organ Cult. 2013, 114, 11-18. [CrossRef]

28. Xu, M.; Chen, C.; Cai, H.; Wu, L. Overexpression of PeHKT1;1 Improves Salt Tolerance in Populus. Genes 2018, 9, 457. [CrossRef]

29. Fukuda, T.; Yokoyama, J.; Nakamura, T.; Song, I.J.; Ito, T.; Ochiai, T.; Kanno, A.; Kameya, T.; Maki, M. Molecular phylogeny and evolution of alcohol dehydrogenase (Adh) genes in legumes. BMC Plant Biol. 2005, 6, 1-10.

30. Strommer, J. The plant ADH gene family. Plant J. 2011, 66, 128-142. [CrossRef] [PubMed]

31. Komatsu, S.; Thibaut, D.; Hiraga, S.; Kato, M.; Chiba, M.; Hashiguchi, A.; Tougou, M.; Shimamura, S.; Yasue, H. Characterization of a novel flooding stress-responsive alcohol dehydrogenase expressed in soybean roots. Plant Mol. Biol. 2011, 77, 309-322. [CrossRef]

32. Li, C.F.; Xu, D.P.; Han, W.B.; Zhang, X.J.; Qi, M.; Tian, C.J.; Zheng, S.F. Genome-wide Identification and Expression Analysis of the ADH Gene Family in Upland Cotton (Gossypium hirsutum L.). Mol. Plant Breed. 2018, 7, 2065-2077.

33. Papdi, C.; Pérez-Salamó, I.; Joseph, M.P.; Giuntoli, B.; Bögre, L. The low oxygen, oxidative and osmotic stress responses synergistically act through the ethylene response factor VII genes RAP2.12, RAP2.2 and RAP2.3. Plant J. 2015, 82, 772-784. [CrossRef] [PubMed] 
34. Drew, M.C. Oxygen Deficiency and Root Metabolism: Injury and Acclimation under Hypoxia and Anoxia. Annu. Rev. Plant Physiol. Plant Mol. Biol. 1997, 48, 223-250. [CrossRef] [PubMed]

35. Shen, C.W.; Yuan, J.P.; Qu, X.Q. Alcohol dehydrogenase (ADH) genes family in wheat (Triticum aestivum): Genome-wide identification, characterization, phylogenetic relationship and expression patterns. Reach Sq. 2020, 8, 1-14.

36. Andrews, D.L.; Cobb, B.G.; Johnson, J.R. Hypoxic and anoxic induction of alcoholdehydrogenase in roots and shoots of seedlings of Zea mays: Adh transcripts and enzyme activity. Plant Physiol. 1993, 101, 407-414. [CrossRef] [PubMed] 